\title{
Prática farmacêutica na seleção e programação de medicamentos no Sistema Único de Saúde (SUS): Revisão de literatura
}

Pharmaceutical practice in drug selection and programming in the Unified Health System (SUS): Literature review

Práctica farmacéutica en la selección y programación de medicamentos en el Sistema Único de Salud (SUS): revisión de la literatura

\section{Resumo}

Introdução: As cadeias de suprimentos de seleção e programação de aquisição de medicamentos público estão se tornando cada vez mais interligadas e estruturadas. Um dos profissionais que atuam nesses processos estão os farmacêuticos, que com suas expertises no manejo de fármacos, contribuem para a eficácia dessas aquisições, no âmbito do SUS. Essas atividades demandam uma percepção analítica e holísticas, para que haja harmonia entre estoque x aquisição x demanda, evitando desperdícios e falta de fármacos. Objetivo: Descrever sobre a importância do farmacêutico nas etapas de seleção e programação de medicamentos, dentro do ciclo da Assistência Farmacêutica no SUS. Metodologia: Trata-se de uma revisão de literatura elaborada com base na busca de dados eletrônicos sendo PubMed, Biblioteca Virtual da Saúde (BVS), Scielo e catálogo do Ministério da saúde. Resultados e Discussão: Identificou-se 09 estudos que serviram de base para o desenvolvimento desta revisão. Os estudos indicam que as atividades de programação e aquisição de medicamentos no âmbito do Sistema Único de Saúde (SUS) demanda um olhar holístico e analítico a fim de equilibrar disponibilidade e quantidade. Conclusão: O eficaz gerenciamento na seleção e programação de medicamentos no âmbito do SUS constitui-se primícias básicas voltadas a busca da promoção da saúde por meio da distribuição do medicamento e do cuidado do dinheiro público. As práticas atuais de seleção e programação de medicamento no SUS tem contribuído para evitar-se desperdício e os dados financeiros e ambientais que o descontrole poderia causar.

Palavras-chave: Atuação do farmacêutico; Sistema único de saúde; Assistência farmacêutica; Uso racional de medicamentos.

\begin{abstract}
Introduction: Public drug procurement scheduling and procurement supply chains are becoming increasingly interconnected and structured. One of the professionals who work in these processes are the pharmacists, who, with their expertise in drug management, contribute to the effectiveness of these acquisitions, within the scope of the SUS. These activities demand an analytical and holistic perception, so that there is harmony between stock $\mathrm{x}$ acquisition $\mathrm{x}$ demand, avoiding waste and lack of drugs. Objective: To describe the importance of the pharmacist in the stages of drug selection and programming, within the cycle of Pharmaceutical Assistance in the SUS. Methodology: This is a literature review based on the search for electronic data such as PubMed, Virtual Health Library (BVS), Scielo and Ministry of Health catalog. Results and Discussion: We identified 09 studies that served as the basis for the development of this review. Studies indicate that drug programming and procurement activities within the Unified Health System (SUS) demand a holistic and analytical look in order to balance availability and quantity. Conclusion:
\end{abstract}


The effective management in the selection and programming of medications within the SUS constitutes basic firsts aimed at promoting health through the distribution of medication and the care of public money. Current practices in the selection and programming of medication in the SUS have contributed to avoiding waste and the financial and environmental data that the lack of control could cause.

Keywords: Pharmacist performance; Unified health system; Pharmaceutical assistance, Rational use of medicines.

\section{Resumen}

Introducción: Las cadenas de suministro de programación y adquisición de medicamentos públicos están cada vez más interconectadas y estructuradas. Uno de los profesionales que trabajan en estos procesos son los farmacéuticos, quienes con su experiencia en la gestión de medicamentos contribuyen a la efectividad de estas adquisiciones, en el ámbito del SUS. Estas actividades exigen una percepción analítica y holística, de manera que exista armonía entre stock x adquisición x demanda, evitando el desperdicio y la falta de medicamentos. Objetivo: Describir la importancia del farmacéutico en las etapas de selección y programación de medicamentos, dentro del ciclo de Asistencia Farmacéutica en el SUS. Metodología: Se trata de una revisión de la literatura basada en la búsqueda de datos electrónicos como PubMed, Biblioteca Virtual en Salud (BVS), Scielo y catálogo del Ministerio de Salud. Resultados y discusión: Identificamos 09 estudios que sirvieron de base para el desarrollo de esta revisión. Los estudios indican que las actividades de programación y adquisición de medicamentos dentro del Sistema Único de Salud (SUS) exigen una mirada holística y analítica para equilibrar la disponibilidad y la cantidad. Conclusión: La gestión eficaz en la selección y programación de medicamentos dentro del SUS constituye primicias básicas encaminadas a promover la salud a través de la distribución de medicamentos y el cuidado del dinero público. Las prácticas actuales en la selección y programación de medicamentos en el SUS han contribuido a evitar el desperdicio y los datos económicos y ambientales que el descontrol podría ocasionar.

Palabras clave: Desempeño farmacéutico; Sistema único de salud; Asistencia farmacéutica; Uso racional de medicamentos.

\section{Introdução}

O Sistema Único de Saúde (SUS) é uma conquista do povo brasileiro e está amparado pela Constituição Federal de 1988, artigo 196, inciso n. 8.080 / 1990. O SUS é o único sistema público de saúde do mundo que atende a mais de 190 milhões de pessoas, e 80\% delas contam exclusivamente com ele para prestar algum atendimento médico". A característica do atendimento medicamentoso é uma série de ações relacionadas à distribuição de medicamentos, tendo como meta a orientação para promover o sucesso do tratamento (dos Santos, da Rosa \& Leite, 2017; Santana, 2017).

O farmacêutico é o profissional que tem as melhores condições para garantir a qualidade dos medicamentos porque a sua formação é direcionada para os medicamentos. Sem um farmacêutico, todo o programa de assistência a medicamentos levará inevitavelmente a um declínio na qualidade (dos Santos, da Rosa \& Leite, 2017).

A Assistência Farmacêutica no uso racional de medicamentos é essencial para melhorar orientação da população e o acompanhamento do uso adequado, dispensação, farmacovigilância, educação a população quanto aos riscos da automedicação, da interrupção e da troca da medicação prescrita (Silva et al., 2016; Rodrigues et al., 2019).

A Política Nacional de Assistência Farmacêutica (PNAF), ao estabelecer a Assistência Farmacêutica desenvolveu um conjunto de ações interligadas envolvendo a pesquisa, o desenvolvimento e a produção de medicamentos e insumos, assim os aspectos que envolvem a sua seleção, programação, aquisição, distribuição, dispensação, assim como a garantia da qualidade dos produtos e serviços, incluindo ainda o acompanhamento e avaliação de consumo.

A programação permite aos gestores de estoques a estimar quantidades a serem adquiridas visando atender as demandas dos serviços disponibilizado pela Unidade de Saúde em função de espaço de tempo determinados. Utiliza-se a Relação Nacional de Medicamentos Essenciais (RENAME) e/ou a Relação Municipal de Medicamentos Essenciais (REMUME), como bases da programação, que após a análise são estabelecidas e confirmadas as quantidades a serem solicitada na etapa de seleção. A RENAME e a REMUME são instrumentos técnico-orientativo com objetivo de promover a promoção do uso racional de medicamentos e para a qualificação da gestão do SUS. 
O planejamento de medicamentos inclui a estimativa da quantidade a ser adquirida para atender às necessidades de um serviço específico em um período de tempo específico. O planejamento inadequado reflete diretamente no fornecimento e acesso de medicamentos.

A importância desse trabalho é que além de orientar as pessoas a forma correta do uso racional de medicamentos e sempre orientar sobre a automedicação e ressaltar a importância do ciclo da Assistência Farmacêutica; a seleção de medicamentos é a base da assistência medicamentosa, pois a seleção de medicamentos proporciona benefícios do tratamento, que estão relacionados ao uso racional dos medicamentos e aos benefícios das soluções de tratamento.

O objetivo desta revisão de literatura foi explicar a importância do farmacêutico nas etapas de seleção e programação de medicamentos do ciclo da Assistência Farmacêutica no SUS e descrever o papel do farmacêutico na fase de seleção e programação de medicamentos no SUS.

\section{Metodologia}

Para a realização desse estudo optou-se por uma pesquisa na modalidade de revisão integrativa de literatura. A revisão integrativa determina o conhecimento atual sobre uma temática específica, já que é conduzida de modo a identificar, analisar e sintetizar resultados de estudos independentes sobre o mesmo assunto (Mendes et al., 2008). Dessa forma, embasada em referências bibliográficas e científicas, visando uma atualização contínua da confiabilidade, uma vez que se desejou verificar os fatores que influenciam a prática farmacêutica na seleção e programação de medicamentos no Sistema Único de Saúde.

Para elaboração da pesquisa, serão realizados busca de dados eletrônicos sendo forma de órgãos oficiais, revistas, artigos em site como SCIELO (Scientifie Eletronic Library) Ministério da Saúde, Resoluções da Anvisa e BVS.

Foram realizadas buscas pelas palavras-chaves (Assistência Farmacêutica, seleção de medicamentos, programação de medicamentos, ciclo da Assistência Farmacêutica e sistema único de saúde). Os artigos foram buscados em português e inglês com o período de tempo estipulado de 10 anos.

No processo inicial da pesquisa retornaram 173 artigos nas bases de dados Scielo (114), BVS (40) e PubMed (19), descartou-se 133 estudos por estarem duplicados.

Dos arquivos rastreados, procedeu-se um comparativo visando identificar os duplicados e estarem de alguma forma fora dos critérios de exclusão adotados nesse estudo, onde resultado no não aproveitamento para constituição dos estudos base foram excluídos 50 estudos por estarem fora do tema, sem metodologia clara ou tratar-se de relato de casos, sendo identificados 83 estudos elegíveis como de uso nesse artigo.

Desses 74 foram excluídos ou por estarem incompletos (10) ou por se tratarem de estudos de casos, com isso, restaram 09 estudos que compõem esse artigo. 
Quadro 1. Corpus do Estudo.

\begin{tabular}{|c|c|c|c|}
\hline AUTOR & $\begin{array}{l}\text { TIPO DE } \\
\text { ESTUDOS }\end{array}$ & TÍTULO & RESULTADO \\
\hline $\begin{array}{l}\text { Perreira, Rebeca } \\
\text { Mancini (2016) }\end{array}$ & Cartilha & $\begin{array}{l}\text { Planejamento, programação } \\
\text { e aquisição: prever para } \\
\text { prover }\end{array}$ & $\begin{array}{l}\text { As atividades de programação e aquisição de medicamentos bem } \\
\text { como os principais aspectos do planejamento que devem ser } \\
\text { considerados para o êxito destas duas atividades relacionadas aos } \\
\text { suprimentos. }\end{array}$ \\
\hline $\begin{array}{l}\text { de Andrade, Luciano } \\
\text { Bezerra (2015) }\end{array}$ & Descritivo & $\begin{array}{c}\text { O papel do farmacêutico no } \\
\text { âmbito hospitalar }\end{array}$ & $\begin{array}{l}\text { Profissional responsável por todo o fluxo do medicamento dentro } \\
\text { da unidade de saúde e pela orientação aos pacientes internos } \\
\text { ambulatoriais, buscando cooperar na eficácia do tratamento, } \\
\text { redução dos custos, voltando-se o ensino e a pesquisa, funcionando } \\
\text { como campo de aprimoramento profissional. }\end{array}$ \\
\hline $\begin{array}{c}\text { Ministério da Saúde } \\
\text { (2006) }\end{array}$ & Revista & $\begin{array}{l}\text { Assistência Farmacêutica na } \\
\text { atenção básica instruções } \\
\text { técnicas para sua } \\
\text { organização }\end{array}$ & $\begin{array}{l}\text { Aplicação dessas informações no âmbito dos serviços do SUS } \\
\text { conferindo maior eficiência no desempenho das ações, servindo de } \\
\text { referenda e de instrumentalização para as atividades da Assistência } \\
\text { Farmacêutica, e contribuindo para a ampliação do acesso da } \\
\text { população aos medicamentos e a promoção do seu use correto. }\end{array}$ \\
\hline $\begin{array}{l}\text { Biscahyno, Francieli \& } \\
\text { Barchet; Limberger, } \\
\text { Jane Beatriz (2013) }\end{array}$ & $\begin{array}{l}\text { Pesquisa } \\
\text { observacional } \\
\text { descritiva, } \\
\text { retrospectiva, } \\
\text { transversal. }\end{array}$ & $\begin{array}{l}\text { Ciclo da Assistência } \\
\text { Farmacêutica e a atuação do } \\
\text { farmacêutico em unidades } \\
\text { básicas de saúde de Santa } \\
\quad \text { Maria - RS }\end{array}$ & $\begin{array}{l}\text { Questionar os participantes sobre as etapas do ciclo da Assistência } \\
\text { Farmacêutica, a saber: seleção, programação, aquisição, armazena- } \\
\text { mento, distribuição e utilização de medicamentos. }\end{array}$ \\
\hline $\begin{array}{c}\text { Ministério da Saúde } \\
\text { (2006) }\end{array}$ & Documento & $\begin{array}{l}\text { Aquisição de medicamentos } \\
\text { para Assistência } \\
\text { Farmacêutica no SUS }\end{array}$ & $\begin{array}{l}\text { Contribuir com a melhoria das compras de medicamentos na rede } \\
\text { púbica de saúde e atender à demanda na área, disponibilizamos este } \\
\text { documento com informações práticas, objetivas e atualizadas, para } \\
\text { todos os profissionais envolvidos com a Assistência Farmacêutica }\end{array}$ \\
\hline $\begin{array}{l}\text { Karnikowski, Margô G. } \\
\text { O., et al (2017) }\end{array}$ & $\begin{array}{l}\text { Estudo } \\
\text { transversal, } \\
\text { exploratório, } \\
\text { de natureza } \\
\text { avaliativa }\end{array}$ & $\begin{array}{l}\text { Caracterização da seleção } \\
\text { de medicamentos para a } \\
\text { atenção primária no Brasil }\end{array}$ & $\begin{array}{l}\text { Os responsáveis da AF relataram não haver Comissão de Farmácia } \\
\text { e Terapêutica formalmente constituída }(12,5 \%) \text {. Eles afirmaram } \\
\text { possuir uma Lista de Medicamentos Essenciais }(85,3 \%) \text { atualizada } \\
(80,4 \%) \text {, e serem participantes deste processo }(88,2 \%) \text {. }\end{array}$ \\
\hline $\begin{array}{l}\text { Patrícia Prevedello, } \\
\text { Maria Assunta Busato }\end{array}$ & Artigo & $\begin{array}{l}\text { Análise do ciclo da } \\
\text { Assistência Farmacêutica } \\
\text { em Romelândia - SC }\end{array}$ & $\begin{array}{l}\text { Melhorias adicionais proporcionando um protocolo de comunicação } \\
\text { mais abrangente (aconselhamento sobre o uso de medicamentos e } \\
\text { implantação de política farmacêutica no município) significariam } \\
\text { que tanto o serviço quanto a sua população poderia trabalhar a } \\
\text { conscientização: o atendimento às ações do ciclo de AF, o público à } \\
\text { medicação necessidades e cursos de terapia para melhorar a } \\
\text { qualidade de vida. }\end{array}$ \\
\hline $\begin{array}{l}\text { Beltrame, A., Regina, } \\
\text { D., Pontarolli, S., \& } \\
\text { Rieck, E. C. M. }\end{array}$ & Livro & $\begin{array}{l}\text { Assistência Farmacêutica } \\
\text { no SUS }\end{array}$ & $\begin{array}{l}\text { A organização da Assistência Farmacêutica, fundamentada no } \\
\text { enfoque sistêmico, caracteriza-se por ações articuladas } \\
\text { sincronizadas entre as diversas partes que compõem o sistema, que } \\
\text { influenciam e são influenciadas umas pelas outras. }\end{array}$ \\
\hline $\begin{array}{l}\text { Cassia, A., Sena, F., \& } \\
\text { Barros, M. }\end{array}$ & Revista & $\begin{array}{l}\text { Gestão de compras em } \\
\text { farmácia hospitalar }\end{array}$ & $\begin{array}{l}\text { As técnicas de normalização são essenciais para um efetivo } \\
\text { planejamento e controle dos medicamentos utilizados nos hospitais }\end{array}$ \\
\hline
\end{tabular}

Fonte: Elaborado pelas autoras.

\section{Resultados e Discussão}

A Assistência Farmacêutica começou com a missão de fornecimento de medicamentos a população sem condições econômicas para adquirir. Com a criação da Lei Orgânica da saúde, no seu artigo $6^{\circ}$ determina a criação da Política de 
Medicamentos o campo de atuação do SUS, no âmbito da Assistência Farmacêutica e as responsabilidades pela execução de ações visando a promoção da Assistência Terapêutica Integral, inclusive farmacêutica (Pereira, 2016).

A concepção mais elaborada da Assistência Farmacêutica deve-se ao Conselho Nacional de Saúde (Resolução CNS nº 338/2004), o qual define a Assistência Farmacêutica como um conjunto de ações voltadas à promoção, proteção e recuperação da saúde, tanto individual como coletivo, destacando que o medicamento é insumo essencial e que deve se priorizar o seu acesso e uso racional. Este conjunto envolve a pesquisa, o desenvolvimento e a produção de medicamentos e insumos, bem como a sua seleção, programação, aquisição, distribuição dispensação, garantia de qualidade dos produtos e serviços, acompanhamento e avaliação de sua utilização, na perspectiva da obtenção de resultados concretos e da melhoria da qualidade de vida da população (Brasil, 2018).

O ciclo da Assistência Farmacêutica é um sistema constituído pelas etapas de seleção, programação, aquisição, armazenamento, distribuição e dispensação, com suas interfaces nas ações de atenção à saúde básica. Para proporcionar os serviços farmacêutica, é preciso que ela esteja estruturada nos âmbitos federal, estadual e municipal. AF por ser uma atividade multidisciplinar e devido a sua complexidade, necessita de articulações permanentes com outras áreas, como vigilância sanitária, epidemiologia, coordenação de programas estratégicos de saúde, programa saúde da família e programa agentes comunitários de saúde. Medicamentos (Biscahyno \& Limberger, 2013).

AF atua agregar ações entre medicamentos e seu usuário, organização mundial de saúde admitir que o farmacêutico e o profissional habilitado para conduzir as ações destinadas a melhoria do acesso a promoção do uso racional de uma aquisição correta de medicamentos não pode faltar o que comprar (Seleção); quando e quanto comprar (programação); e como comprar (Karnikowski et al., 2017).

A seleção de medicamentos é um processo muito importante que consiste na escolha do medicamento dentre todos os itens referendados pela Relação Nacional de Medicamentos Essenciais, tendo como critério principal o perfil epidemiológico, econômico e técnico (de Andrade, 2015).

Nos níveis de atenção à saúde, a Comissão de Farmácia e Terapêutica exibe um papel estratégico na seleção de medicamentos, com o propósito de garantir segurança, eficácia e custo-efetividade no Sistema Único de Saúde, tendo como objetivo estabelecer as condutas terapêuticas e direcionar o processo de aquisição (Brasil, 2018).

A seleção de medicamentos é importante, pois a partir dela são estabelecidas as etapas da Assistência Farmacêutica (Cortez, Cortez \& Leite, 2014; Abreu et al., 2020). Com raras exceções tal como ocorre em organizações de outras setores há necessidade de se controlar bem os estoques adquiridos, principalmente de medicamentos e insumos que possuem alto consumo, custo e volume, como forma de se controlar adequadamente a reposição desses.

Esse processo de gestão de estoque são armadilhas presentes na tomada das decisões de quanto pedir, quando pedir e quanto manter em estoques de segurança (Rocha et al., 2019).

Até os anos 1980 as adotava-se os métodos de equivalência para gerir os estoques de medicamentos, processo esses que geravam falhas na seleção e aquisição de medicamento, onde se observava grande desperdícios de medicamentos e insumo e em geral os custos com aquisição, estoque e descarte era relegado a segundo plano salientam ainda os autores Maiellaro et al., (2017).

A partir de 1990, quando novas técnicas de gestão de estoque e o fortalecimento da Assistência Farmacêutica, métodos como curva $\mathrm{ABC}$ trouxe uma visão na perspectiva do estoque como um elemento muito importante que pode influenciar na eficácia da gestão do estoque (Corsato et al., 2016).

Ao contrário do que se possa pensar, a má gestão de estoque pode causar danos significativos a saúde do usuário pode ser do tipo: Estoque insuficiente: a unidade não consegue atender pedidos e, portanto, perda de dados e credibilidade e 
Excesso de estoque: alto custo de armazenamento sem esquecer que o estoque pode se deteriorar neste período (Biscahyno \& Limberger, 2013).

Portanto, é importante manter um nível de estoque de acordo com a estratégia do RENAME, uma vez que o estoque é antes de tudo um ativo importante para a promoção da saúde, sendo esse uma das atividades de farmacêuticos por meio de auditorias e balanços reajustar, avaliar, (re) programar solicitar os medicamentos e insumos no âmbito do SUS, tal como ocorre na iniciativa privada (Maiellaro et al, 2014).

Existem várias ferramentas para gerir o estoque, cujo cálculo é importante para o administrador ter visibilidade do seu os custos. Com isso, a seleção de medicamentos e a fase mais importante do ciclo, é o processo de escolha de medicamentos eficazes e seguros necessários ao atendimento das necessidades de uma população, a seleção de medicamentos essenciais tende a enriquecer a qualidade da prescrição e favorecer o processo da escolha dos medicamentos (Ferreira \& Soller, 2021).

Para Menegat (2018), a seleção de medicamentos e o eixo orientador parar as ações de planejamento e de organização da Assistência Farmacêutica, se bem administrada conquista ganhos econômicos para o Sistema Único de Saúde (SUS).

Para ocorrerá formar adequada da seleção ela deve cumpriu algumas formalidades, como a implantação da Comissão de Farmácia e Terapêutica (CFT), cuja obrigação e a condução técnica, política e administrativa do processo. O fundamental produto desse processo é uma Lista de Medicamentos Essenciais (LME), tem base na pesquisa epidemiológica, com critério de proporcionar medicamentos prioritários a saúde pública, eficazes, seguros, qualidade assegurada, prescritos e usados racionalmente (Monteiro et al., 2017).

A seleção deve ser feita por profissionais da saúde, com conhecimentos, fatores e habilidades. O uso deve ser aplicado obrigatoriamente nos serviços de saúde e principalmente por quem prescreve. Deve conferir frequentemente e comunicar aos profissionais de saúde (Medeiros, 2018).

Outras informações essenciais a seleção e aquisição de medicamentos, refere-se ao fato que todos os medicamentos devem atender o perfil epidemiológico local e estarem contextualizados com as realidades locais de atendimento das principais dificuldades da saúde (Carvalho \& Neto, 2018).

O objetivo principal é determinar o total de medicamento escolhido que deve ser adquirido e priorizado com meios à disposição de evitar a suspensão do fornecimento. Avaliação da demanda programada deve levar em conta a circunstância do local de saúde, o acesso dos pacientes com os medicamentos, a descrição de doenças da população, propostas de serviços, protocolos clínicos e a flexibilidade do orçamento e financeiro (Abreu et al., 2020).

A elaboração da programação estabelece medidas de recebimento do produto: a data de fabricação até a data de entrega ao almoxarifado ao destino, não pode ter passado mais de $20 \%$ do prazo da validade, embalagem em excelente estado, situações de armazenamento, transporte com as especificações do produto (calor, luz, temperatura e umidade), a presença do laudo de controle na entrega do produto, cópia autenticada da licença de funcionamento e copia autenticidade pelos seguintes documentos exigidos pela Anvisa: certificado de boas práticas de distribuição e armazenamento de medicamentos válido, registro válido do medicamento (São Paulo, 2020).

Há ainda as questões que envolvem a autorização de funcionamento do fabricante, autorização especial do fabricante se o medicamento for sujeito a controle especial (Portaria $n^{\circ}$ 344/98) e certificado de boas práticas de fabricação e controle de medicamentos (Brasil, 2018).

\section{Conclusão}

Conclui-se que o gerenciamento de estoque no âmbito do SUS é um processo complexo, mas crítico dentro do sistema de prestação de serviços de saúde. Sem práticas adequadas de gerenciamento de estoque farmacêutico, as unidades de saúde correm o risco de não conseguir fornecer aos pacientes os medicamentos mais adequados quando eles são mais necessários. 
O farmacêutico desempenha um importante papel, não somente no planejamento e aquisição desses medicamentos, mas nos aspectos que envolve os padrões de distribuição das farmácias e as opções de seleção de medicamentos podem ter um efeito direto sobre a acessibilidade do atendimento, identificação e relato de recebimento de medicamentos não contratados ou não incluídos RENAME e REMUME, contribuindo assim para redução de custos, disponibilidade de estoque, evitando assim o desperdício de medicamentos.

\section{Referências}

Abreu, R. D. S., et al. (2020). Assistência farmacêutica em unidades básicas de saúde: um foco no serviço farmacêutico. Brazilian Journal of Health Review, 3(4), 9897-9911.

Biscahyno, F. B., \& Limberger, J. B. (2013). Ciclo da assistência farmacêutica e a atuação do farmacêutico em unidades básicas de saúde de Santa Maria-RS. Infarma-Ciências Farmacêuticas, 25(1), 43-50.

Carvalho, C. C. A., \& Neto, O. H. C. (2018). Papel do profissional farmacêutico no Sistema Único De Saúde (SUS) em um município de Minas Gerais. Revista Brasileira de Ciências da Vida, 6(3).

Corsato, R. F., et al. (2016). Contribuição do método curva ABC para gestão das farmácias hospitalares. Revista Conexão Eletrônica, 13(1).

Cortez, D. X., Cortez, F. O. X., \& Leite, R. M. (2014). Assistência farmacêutica no SUS. Revista Interfaces: Saúde, Humanas e Tecnologia, 2(5).

de Andrade, L. B. (2015). O papel do farmacêutico no âmbito hospitalar.

Dos Santos, V. B., da Rosa, P. S., \& Leite, F. M. C. (2017). A importância do papel do farmacêutico na Atenção Básica. Revista Brasileira de Pesquisa em Saúde/Brazilian Journal of Health Research, 19(1), 39-43.

Ferreira, R. L., \& Soler, O. (2021). Prática de governança e gestão técnica da assistência farmacêutica no Município de Xinguara, Pará. Research, Society and Development, 10(4), e51210414416-e51210414416.

Karnikowski, M. G. O., et al. (2017). Caracterização da seleção de medicamentos para a atenção primária no Brasil. Revista de Saúde Pública, 51.

Maiellaro, J. R., et al. (2014). A gestão de estoques de medicamentos: Um estudo de caso em um hospital público. INOVAE-Journal of Engineering, Architecture and Technology Innovation (ISSN 2357-7797), 2(1), 18-33.

Medeiros, A. L. (2018). Assistência farmacêutica no Sistema Único de Saúde: responsabilidade compartilhada por União, Estado e Municípios. Tese de Doutorado. Universidade de São Paulo.

Mendes, K. D. S., Silveira, R. C. C. P., \& Galvão, C. M. (2008). Revisão integrativa: método de pesquisa para a incorporação de evidências na saúde e na enfermagem. Texto Contexto Enferm, 17(4), 758-64.

Menegat, F. (2018). O farmacêutico na gestão da política nacional de medicamentos: revisão de literatura.

Ministério da Saúde. Secretaria de Ciência, Tecnologia e Insumos Estratégicos. Departamento de Assistência Farmacêutica e Insumos Estratégicos. Assistência Farmacêutica no SUS: 20 anos de políticas e propostas para desenvolvimento e qualificação - relatório com análise e recomendações de gestores, especialistas e representantes da sociedade civil organizada. Brasília: Ministério da Saúde.

Monteiro, A. S., et al. (2017). A Logística na Aquisição de Medicamentos pelo SUS no Município de Petrolina. ID on line Revista de Psicologia, 11(37), 3745 .

Pereira, R. M. (2016). Planejamento, Programação e Aquisição: prever para prover. Organização Pan-Americana da Saúde. Série Uso racional de medicamentos: fundamentação em condutas terapêuticas e nos macroprocessos da Assistência Farmacêutica, 1(10).

Rocha, W. H., et al. (2019). Fatores que podem influenciar a disponibilidade dos medicamentos essenciais na atenção primária à saúde (APS) do Sistema Único de Saúde (SUS).

Rodrigues, R. M. L., et al. (2019). O papel do farmacêutico na assistência farmacêutica com ênfase na orientação quanto ao uso racional de medicamentos.

Santana, K. Dos S. (2017). O papel do profissional farmacêutico na promoção da saúde e do uso racional de medicamentos.

São Paulo. (2020). Secretaria Municipal de Saúde de São Paulo. Descrição de Atribuições e Técnicos de Farmácia. São Paulo: Secretaria Municipal de Saúde de São Paulo.

Silva, A. O. M., et al. (2016). O papel do farmacêutico na automedicação de medicamentos isentos de prescrição. Revista de Trabalhos Acadêmicos-Campus Niterói, 2. 